\title{
Wnt5a Role in Cisplatin Effect and Selectivity Augmentation in Cancer Therapy
}

\author{
Waleed 0. Atta' ${ }^{1}$ Ahmed Z. Abdel Azeiz ${ }^{*}$ \\ ${ }^{1}$ Faculty of Medicine, Assiut University, Sohag, Egypt \\ ${ }^{2}$ College of Biotechnology, Misr University for Science and Technology (MUST), 6th of October, Egypt \\ Email: *ahmed.abdelaziz@must.edu.eg
}

How to cite this paper: Atta, W.O. and Azeiz, A.Z.A. (2021) Wnt5a Role in Cisplatin Effect and Selectivity Augmentation in Cancer Therapy. Open Access Library Journal, 8: e7564.

https://doi.org/10.4236/oalib.1107564

Received: May 21, 2021

Accepted: June 25, 2021

Published: June 28, 2021

Copyright $\odot 2021$ by author(s) and Open Access Library Inc.

This work is licensed under the Creative Commons Attribution International License (CC BY 4.0).

http://creativecommons.org/licenses/by/4.0/

(c) (i) Open Access

\begin{abstract}
Wnt5a is a representative protein ligand that activates a beta catenin independent pathway in Wnt signaling in cancer progression and spread in the palmitoylated form. So that its synthesis is augmented through promotion of its gene transcription in the malignant cells. Cisplatin is an important chemotherapeutic drug used in treatment of different types of advanced cancer. However, its usage is limited due to severe dose-limiting side effects, due to the lack of targeting therapy of cisplatin. On the other hand, the cancer cells developed resistance from cisplatin that is solved partially by C-glycosylation of cisplatin. Through this review article, we strongly suggest that the Wnt5a protein can be used as drug delivery molecule for cisplatin to target the malignant cells. This can be performed through the following steps: Firstly: use of Wnt5a in the depalmitoylated form to avoid its effect on cancer progression with improvement of its binding efficacy by two suggested modifications: 1) Glycosylation with sialic acid-containing glycoside to increase the binding affinity with the sialic acid on the surface of the malignant cells; 2) GPI anchoring modification: This can be performed through the Serine 282 in the Wnt5a molecule. Secondly, binding of the C-glycosylated cisplatin molecule with the modified Wnt5a molecule through the C-glycosylation bond. Since one Wnt5a molecule contains four asparagine molecules, it can bind with four molecules of C-glycosylated cisplatin molecules, hence higher concentration of cisplatin will attack one malignant cell.
\end{abstract}

\section{Subject Areas}

Biochemistry, Bioengineering, Biotechnology

\section{Keywords}

Cisplatin, Malignant Cells, Wnt5a 


\section{Introduction}

\subsection{Cisplatin as a Therapeutic Agent against Cancer}

Cisplatin (Figure 1) is an alkylating anti-neoplastic drug that is acting by adding alkyl group to many electronegative groups under conditions present in the cell. Cisplatin, after entering the cell, stops the tumor growth by cross-linking guanine bases by platinum in DNA double helix strands. It causes DNA damage by distorting DNA to prevent it from being separated. It mispairs the nucleotides [1]. Cisplatin is the main treatment of small cell lung cancers, metastatic ovarian cancer, head and neck carcinoma and breast cancer [2].

Cisplatin platinum (II) has made big success but its dosage was limited due to side effects that appeared due to the accumulation of platinum (II) in the healthy tissues such as nephrotoxicity, myelosuppression, and ototoxicity. Platinum (IV) compounds are characterized by its selective and active antitumor activity via reduction to platinum (II) complexes inside cancer cells by reductants like ascorbic acid and glutathione, which are present in higher concentrations in the tumor cells than in normal tissue and blood, improving their direction effect towards malignant cells. By platinum (IV) compounds, metallic platinum molecules will be of less concentration in healthy tissues [3]. Cisplatin can be augmented more in selectivity and efficacy to cancer cells and less side effects to healthy tissue. This can be done if it is glycosylated with sialic acid-containing glycoside.

\subsection{C-Glycosylation of Cisplatin}

Sugar-platinum conjugates (Figure 2) have been confirmed as enzyme activating compounds that have a major role in antibody-directed enzyme prodrug therapy approach [4] [5]. The system aims to restrict the action of high concentration of cytotoxic drug to cancer sites by using antibody (or specified molecule) to<smiles>N[PH](N)(Cl)Cl</smiles>

\section{Cisplatin}

Figure 1. Cisplatin structure.

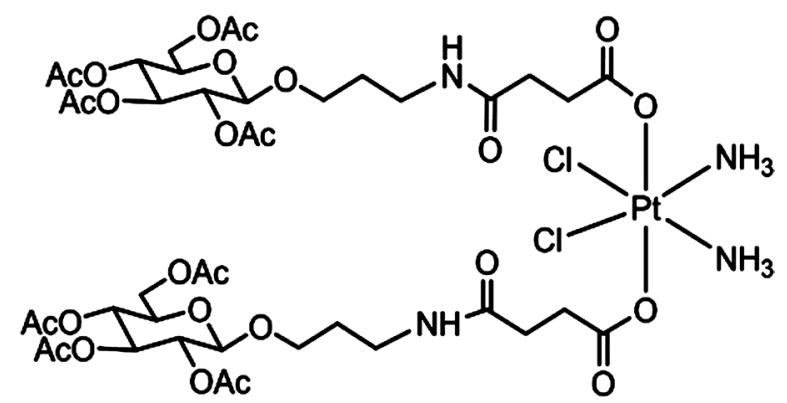

Figure 2. C-glycosylated Cisplatin. 
deliver a non-humanized enzyme to cancer sites. Glycosylation of platinum (IV) prodrugs surmounts the high side-effects of cisplatin-based chemotherapy after activating them to big extent in malignant cells rather than in normal cells by reductants previously mentioned [3]. Cisplatin analogues such as oxoplatin, oxaliplatin and their glycosylated forms also are characterized by broad and intensified antitumor activity [3].

\subsection{Cisplatin Resistance Mechanisms}

1) Reduction in cisplatin influx: Copper transporters are the main gates in the entry and the export of platinum-based agents such as Cu-transporter 1 (CTR1) that are impressive in the cellular uptake of cisplatin [6] [7] [8] [9]. Cervical cancer cells, which are resistant to platinum based drugs, show low expression of CTR1 [6] [7] [8] [9]. Copper transporter 2 (CTR2) primarily situated in intracellular vesicles. CTR2 cleaves CTR1 leading to reduced influx of cisplatin [10]. Patients with elevated expression of CTR2 in ovarian cancer had poor prognosis compared with those of low expression levels [11] [12]. Organic cation transporter 2 (OCT2) helps the cell to gain cisplatin intracellularly. Malignant cells that have high expression of OCT2 respond more positively to cisplatin [11] [12].

2) Increase in cisplatin efflux: Copper transporting ATPase 1 and 2 (ATP7A and B) induce elimination the excess copper from cells, in another words they antagonize CTR1 action. Cervical cells resistant to platinum drug show elevated expressions of ATP7A [9]. Also multidrug resistance associated proteins MRPs especially MRD2 can resist cisplatin (II) through elimination of platinum GSH conjugates (Platinum-glutathione conjugation, Cisplatin (platinum II) reduction by glutathione). Increased levels of MRD2 are related to worse results to cisplatin with colorectal, hepatocellular and esophageal cancer [13] [14].

3) Intracellular drug detoxification: Formation of platinum-GSH conjugates decrease the activity of cisplatin, besides they predispose to its excretion through the action of MRPs. So that it causes drug resistance. Moreover, MTs has important role in operation the cellular metal homeostasis and detoxification upon exposure to heavy metal. Studies have shown that cisplatin-resistant tumor cells have high expression levels of these detoxification enzymes [15] [16].

4) Nucleotide excision repair: After binding to DNA, most of CDDP-induced DNA damage is included in the formation of intra-strand platinum-DNA adducts [17] [18]. Malignant cells can save itself by the help of at least 30 proteins that can discover, certify, unwind, and remove DNA adducts and the filling of resulted gaps after the excision of platinum DNA complexes. Among these NER-associated proteins, there is a DNA excision repair protein ERCC-1 (ERCC1) that removes platinum adducts through ERCC1-DNA repair endonuclease XPF. Several studies have assessed the role of ERCC1 in cancer patients receiving CDDP-based therapies [18]. Various reports confirmed that CDDP efficacy is decreased with high levels of ERCC-1 in patients with ovarian [19] [20], lung [20] [21], nasopharyngeal [22], esophageal [23], cervical [24], head and 
neck [25] and biliary tract cancer [26].

5) HSPs (Heat shock proteins): These are responsible for protein denaturation and have significant roles in cisplatin resistance. For example, HSP 60 is significantly high in cervical and liver carcinoma [27]. Elevated levels of HSP27, HSP70, HSP72, GRP78 and HSP 90 are included in chemotherapy resistant ovarian cancer [28] [29], breast cancer [30], colon cancer [31], cervical HeLa cells [32] and laryngeal carcinoma cells [33].

6) Low molecular weight GTP-binding proteins of Rho family (RhoA, RhoB. RhoC and Rac that belongs to the Ras tumour suppressor gene family: Rac 1 (Rho GTPase), is involved in head and neck squamous cell carcinoma insensitivity to cisplatin [34]. Low expressed level of small GTPase such as Rab5, Rac 1 and RhoA was observed in human cisplatin resistant cells [35].

7) Transcriptional factors: Y-box binding protein-1, CCAAT-binding transcriptions factor2, activating transcription factor 4, zinc finger factor 134, the nuclear transcription factor- $\mathrm{B}$, the microphthalmia-associated transcription factor, the forkhead transcription factor $\mathrm{O}$, and mitochondrial transcription factor A have the ability to resist cisplatin [27].

8) Nuclear factor (Erythroid-Derived 2)-like ( $\mathrm{Nr}-\mathrm{f} 2)$ : The expression of this factor in cancer cell line is associated with cisplatin resistance. Also increased its expression in GCF2-transfected KB3-1 cells reduces cisplatin accumulation resulting in 3-fold resistance to cisplatin [27].

\section{Wnt5a Protein}

Wnt5a is a representative protein that activates cancer progression and spread in the palmitoylated form through different mechanisms. The roles of the post-translational modification in the actions of wnt5a were previously investigated. Wnt5a is modified with palmitate at Cys104 and glycans at Asn114, Asn120, Asn311 and Asn325. The palmitoylation is not essential for the secretion of Wnt5a but it is necessary for its ability to stimulate cell migration. Glycosylation was necessary for the secretion of Wnt5a but not essential for actions of Wnt5a [36].

\subsection{Wnt5a Role in Malignancy}

Wnt5a expression induces proliferation of many cancer types, including glioma, lung cancer and T-cell leukemia, breast,cerebral, gastric and prostatic cancer. Wnt5a is expressed in $30 \%$ in cases of prosatae cancer. However, knockdown of wnt5a did not affect gastric or prostatic malignant cell proleferation in-vivo or in-vitro [36]. Anti-wnt5a inhibits tumor metastasis but not the proliferation of gastric cancer cells in-vivo [37]. So, wnt5a is inevitable cause of cancer metastasis and proliferation, also it has great affinity to different types of cancer cells.

\subsection{Use of Wnt5a as a Drug Delivery Molecule}

Through this review we suggest to use Want5a protein as a drug delivery carrier 
for cisplatin. Wnt5a is a carcinogenic molecule in its palmitoylated form, it will be dangerous to use it in its palmitoylated form, at the same time we need to preserve its affinity to cancer cells, so we suggest to compensate the affinity of palmitoylated wnt5a to cancer cells by GPI anchoring and sialylation of its glycosylated form Figure 3.

\subsection{Steps of Using the Wnt5a Protein as a Drug Delivery Molecule}

From our point-of-view, Wnt5a protein can be used as a drug delivery molecule for anticaner compounds through three steps:

1) Glycosylation and sialylation of depalmitoylated Wnt5a.

2) GPI anchoring of depalmitoylated Wnta5a.

3) Attachment of chemotherapuetic agent to the modified Wnt5a.

\subsection{Glycosylation Wnt5a Protein}

Polysialic acid chains (PSA) form a helical structure of $a$ 2-8-linked sialic acid residues of more than 55 monomers and generate a highly negative charged moiety [38] [39] [40] [41]. PSA influences signal transduction action, because it has the ability to modify intercellular interactions. It has also the advantage to act as a receptor itself [42] [43]. Fucose can exist as a terminal modification or serve as an attachment point for adding other sugars used for repeating $\mathrm{N}$-acetylglucosamine [44]. The PSA chain can be attached to the protein molecule through different types of glycans. Through this hypothesis glycosylated wnt5a can be sialylated.

\section{Role of wnt5a Sialylation}

Sialic acid based glycol-conjugates coat surfaces of many different cell types; also it has a major role in mediating, binding and influx to different families of viruses [45]. So sialylation of wnt5a will augment its binding to different sialic acid-based structures on platelets and leucocytes.

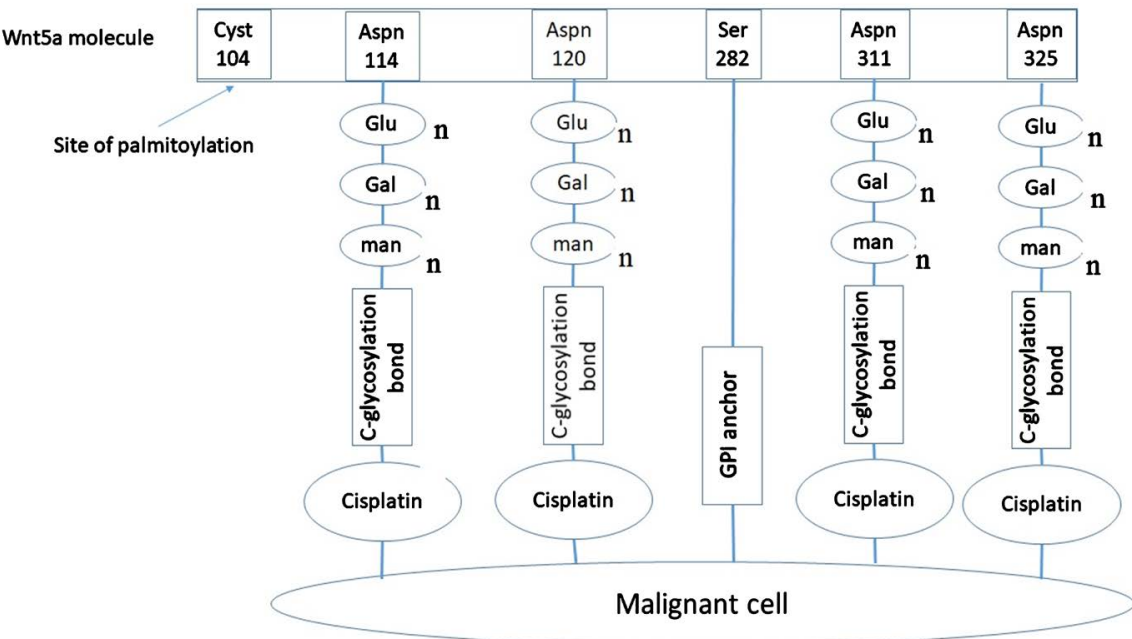

Figure 3. The proposed use of Wnt5a as a drug delivery molecule for cisplatin. 


\subsection{Glycan Structures Candidates for Carrying PSA Chains}

1) $\left((\mathrm{Gal})_{1}(\mathrm{GlcNAc})_{3}(\mathrm{Man})_{4}(\mathrm{fuc})_{1}\right)$ : This glycan contains unusual Ga1 $\beta 1-3$ structure, which is working as a carrier of PSA. This allowed possible substitution of the outer arm G1cNac with $\mathrm{N}$-acetylneuraminic acid (NeuNAc) (a2-6-linked) as well as the galactose residue itself.

2) $\left((\mathrm{Gal})_{2}(\mathrm{GlcNAc})_{6}(\mathrm{Man})_{3}(\mathrm{Fuc})_{2}\right)$ : This compound always observed in sialylated state. More than $90 \%$ possessed a proximal $\alpha 2-6$ linked sialic acid, $10 \%$ have $\alpha 2$-3/8-linked sialic acid residues.

3) $\left((\mathrm{Gal})_{2}(\mathrm{GlcNAc})_{6}(\mathrm{Man})_{3}(\mathrm{fuc})_{1}\right)$ : This compound has ratio of sialylated to non-sialylated form 6:1, possibility of $\alpha 2-6$ linked sialic acid to the outer arm G1cNAc.

\subsection{Wnt5a Sialylation Enzymes}

PSA is biosynthesized by different polysialtransferases. Three different enzymes can attach PSA to G1cNAc, the polysialytransferases ST8SiaII/STX, ST8SiaIV/ PST, which show different spatial and temporal expression, and ST8SiaIII [38].

\subsection{GPI Anchoring of Wnt5a Molecule}

Anchoring of GPI will be at Serine 282 of the Wnt5a molecule. GPI anchoring will compensate the defective affinity of depalmitoylated Wnt5a. This can be achieved by genetic engineering that has the advantage of being conducted in the field of tumor immune therapy. In a previous investigation, a plasmid construct encoding GPI-anchored IL2 was delivered by lipofection to the murine melanoma cell line [46]. The modified murine melanoma cells were investigated for their immune-stimulatory potential. The modified cells were used as a tumor cell vaccine on mice. It reduced the tumor size and prolonged the survival.

GPI signaling sequences (GSS) in a protein are the needed items for GPI anchoring. The GSS contains the later site of GPI attachment (the amino acid in $\omega$ position like Ala, Asn, Asp, Cys, Gly or Ser) and a series of hydrophobic amino acids, essentially forming a membrane associating domain linking the pre-GPI protein to the luminal side of endoplasmic reticulum.

\subsection{Wnt5a-Cisplatin Structure}

The glycosylated asparagine residues on wnt5a Asn 114, 120, 311, 325 can bind to 4 molecules of cisplatin (Cisplatin is C-glycosylated with branch glycosides). By that way, sialic acids present on glycosylated wnt5a will be preserved for binding malignant cells besides GPI anchors (Figure 3). Also wnt5a molecule is familial to different malignant cells that is evidenced by its increased production though promotion its genetic sequence insides the nucleus, so it will be easily recognized by malignant cells other than normal cells. Wnt5a will be safe not functioning after depalmitoylation, just a carrier to platinum prodrug in higher concentration. By that way resistance mechanisms of cisplatin will be reduced as illustrated below: 
1) Improved stability, increased half-life time in biological fluids by c-glycosylation of cisplatin (platinum IV prodrug).

2) Adjusting the length and steric hindrance of axial ligands by $\mathrm{C}$-glycosylation and sialylation to accommodate the reduction potential of malignant cell enzymatic system with a positive shift in cancer cells but not normal cells, especially after binding to modified Wnt5a through lipophilicity of GPI anchor. This can increase the ability to enter tumor cells before being reduced to the active Pt (II) in the circulation (blood).

3) Sialic acids that are present on glycosylated Wnt5a will increase the bioavailability throughout the whole body tissue in case of binding to selectins (single chain transmembrane glycoproteins, which bind to sugar moieties, also it has role in cancer progression). Tumor cells exploit the selectin-dependent mechanisms mediating cell tethering and rolling interactions through recognition of carbohydrate ligands on tumor cell to enhance distant organ metastasis showing "leucocyte mimicry" that are present on migrating leucocytes, platelets and endothelial cells to distant tissues and organs. So, cisplatin can reach to distant organs when carried on the polysialylated wnt5a that bind with Platelet or endothelial selectins. By that way Wnt5a-selectin complexes will augment cisplatin delivery to distant metastatic cancer cells by the same mechanism of cancer spread (binding with selectins). By the same mechanism, selectins will be occupied by binding to sialylated Wnt5a proteins that carry cisplatin, so malignant cells will have limited number of selectins to bind with, by that way its mechanism for spread is limited, in result distant spread will be reduced.

4) Effect on cisplatin resistance mechanisms:

a) Cisplatin carried after C-glycosylation and carried on modified Wnt5a will make another way for cisplatin for reaching and entry malignant cells other than CRT1 (responsible for influx) even if its level on surface membrane is suppressed, also bypassing the effect of CRT2 (responsible for degradation CRT1) after bypassing CRT1.

b) Copper transporting ATPase 1 and 2 (ATP7A and B), which remove excess copper from cells: modified form will avoid that mechanism also as cisplatin depends on the modified wnt5a affinity to malignant cells not copper.

c) Intracellular drug detoxification: C-glycosylation will enable cisplatin to regain its antitumor activity via reduction platinum (IV) to platinum (II) intracellularily, instead of reduction cisplatin (unmodified form) to platinum-GSH conjugates (GSH conjugates is the main mechanism for detoxification).

d) MRD2 (mediates resistance to CDDP through removal platinum GSH conjugates). There is no platinum GSH conjugates to be removed.

e) Nucleotide excision repair: In the hypothesis that one molecule of wnt5a-cisplatin molecule binding to malignant cell, there will be 4 platinum adducts to malignant cell DNA after entry the modified wnt5a-cisplatin (that contain four C-glycosylated cisplatin molecules) which may overcome the ability of ERCC1 to repair the platinum induced DNA damage. In the possibility of mul- 
tiple wnt5a-cisplatin molecules binding, the number of platinum-DNA adducts will be duplicated four times. For example, if two molecules of wnt5a-cisplatin bind the malignant cell, there will be eight platinum DNA adducts, four molecules will be resulted in 16 DNA-platinum adducts, and so on. So we can imagine the number of wn5a-cisplatin molecule binding affinity to malignant cells especially after the suggested modifications, it can result in a lot of DNA-platinum adducts that Nucleotide excision repair of cancer cell could be disabled to save its DNA from the platinum attack towards its DNA.

\section{Conclusions}

Wnt5a is a representative protein ligand that activates cancer progression and spread in the palmitoylated form, while in the depalmitoylated form it loses its carcinogenic effect. Cisplatin is an important chemotherapeutic drug used in treatment of different types of cancer. The glycoside derivatives of cisplatin are more effective. However, its usage is limited due to severe dose-limiting side effects, as well as the resistance mechanisms developed by the tumor cell.

Through this review, it was suggested that the depalmitoylated Wnt5a protein can be used as a drug delivery molecule for the chemotherapeutic agent of cancer such as cisplatin. The depalmitoylated Wnt5a molecule can be modified to improve its binding efficiency with the cancer cell by increase its lipophilicity via GPI anchoring from the Ser 282 residue. Furthermore, it can be glycosylated at four asparagine molecules (Aspn 114, 120, 311, 325) by sialic acid containing glycoside. These modifications will increase Wnt5a bioavailability and attachment to cell membrane (compensation the lipophilic effect of palmitoylation). The Wnt5a glycosylation enables this molecule to bind with four cisplatin molecules. In other words, when wnt5a attaches malignant cell it can deliver four cisplatin molecules intracellular which will have more efficacy. So, Wnt5a will act as safe transporter (depalmitoylated form) with four sialic acid molecules at its termination and GPI anchor molecule, as Wnt5a is recognized easily by malignant cells. In addition, Wnt5a will eliminate many resistance mechanisms of cancer cells towards cisplatin.

\section{Conflicts of Interest}

The authors declare no conflicts of interest regarding the publication of this paper.

\section{References}

[1] Garcia Sar, D., Montes-Bayon, M., Aguado Ortiz, L., Blanco-Gonzalez, E., Sierra, L.M. and Sanz-Medel, A. (2008) In Vivo Detection of DNA Adducts Induced by Cisplatin Using Capillary HPLC-ICP-MS and Their Correlation with Genotoxic Damage in Dorsophilia melanogaster. Analytical and Bioanalytical Chemistry, 390, 37-44. https://doi.org/10.1007/s00216-007-1634-z

[2] Bahadır, A., Ceyhan, A., Öz Gergin, Ö., Yalçın, B., Ülger, M., Özyazgan, T.M. and Yay, A. (2018) Protective Effects of Curcmin and Beta Carotene on Cispla- 
tin-Induced Cardiotoxicity: A Experimental Rat Model. The Anatolian Journal of Cardiology, 19, 213-221. https://doi.org/10.14744/AnatolJCardiol.2018.53059

[3] Ma, J., Wang, Q., Yang, X., Hao, W., Huang, Z., Zhang, J., Wang, X. and Wang, P. (2016) The Glycosylated Platinum(IV) Prodrugs Demonstrated Significant Therapeutic Efficacy in Cancer Cells and Minimized Side-Effects. Dalton Transactions, 45, 11830-11838. https://doi.org/10.1039/C6DT02207C

[4] Tromp, R.A., Stella, S.G., Marco, Timmers, C., van Zutphen, S., van der Marel, G.A., Overkleeft, H.S., van Boom, J.H. and Reedijk, J. (2004) The Beta-Glucuronyl-Based Prodrug Strategy Allows for Its Application on Beta-Glucuronyl-Platinum Conjugates. Bioorganic \& Medicinal Chemistry Letters, 14, 4273-4279. https://doi.org/10.1016/j.bmcl.2004.06.015

[5] Deng, D., Xu, C., Sun, P., Wu, J., Yan, C., Hu, M. and Yan, N. (2014) Crystal Structure of the Human Glucose Transporter GLUT1. Nature, 510, 121-125. https://doi.org/10.1038/nature13306

[6] Amable, L. (2016) Cisplatin Resistance and Opportunities for Precision Medicine. Pharmacological Research, 106, 27-36. https://doi.org/10.1016/j.phrs.2016.01.001

[7] Chen, S.J., Kuo, C.C., Pan, H.Y., Tsou, T.C., Yeh, S.C. and Chang, J.Y. (2015) Mechanistic Basis of a Combination D-Penicillamine and Platinum Drugs Synergistically Inhibits Tumor Growth in Oxaliplatin-Resistant Human Cervical Cancer Cells in Vitro and in Vivo. Biochemical Pharmacology, 95, 28-37. https://doi.org/10.1016/j.bcp.2015.03.006

[8] Chen, S.J., Kuo, C.C., Pan, H.Y., Tsou, T.C., Yeh, S.C. and Chang, J.Y. (2016) Desferal Regulates hCtr1 and Transferrin Receptor Expression through Sp1 and Exhibits Synergistic Cytotoxicity with Platinum Drugs in Oxaliplatin-Resistant Human Cervical Cancer Cells in Vitro and in Vivo. Oncotarget, 7, 49310-49321. https://doi.org/10.18632/oncotarget.10336

[9] Lai, Y.H., Kuo, C., Kuo, M.T. and Chen, H.H.W. (2018) Modulating Chemosensitivity of Tumors to Platinum-Based Antitumor Drugs by Transcriptional Regulation of Copper Homeostasis. International Journal of Molecular Sciences, 19, Article No. 1486. https://doi.org/10.3390/ijms19051486

[10] Öhrvik, H., Logeman, B., Turk, B., Reinheckel, T. and Thiele, D.J. (2016) Cathepsin Protease controls Copper and Cisplatin Accumulation via Cleavage of the Ctr1 Metal-Binding Ectodomain. Journal of Biological Chemistry, 291, 13905-13916. https://doi.org/10.1074/jbc.M116.731281

[11] Lee, Y.Y., Choi, C.H., Do, I.G., Song, S.Y., Lee, W., Park, H.S., Song, T.J., Kim, M.K., Kim, T.J., Lee, J.W., et al. (2011) Prognostic Value of the Copper Transporters, CTR1 and CTR2, in Patients with Ovarian Carcinoma Receiving Platinum-Based Chemotherapy. Gynecologic Oncology, 122, 361-365.

https://doi.org/10.1016/j.ygyno.2011.04.025

[12] Yoshida, H., Teramae, M., Yamauchi, M., Fukuda, T., Yasui, T., Sumi, T., Honda, K. and Ishiko, O. (2013) Association of Copper Transporter Expression with Platinum Resistance in Epithelial Ovarian Cancer. Anticancer Research, 33, 1409-1414.

[13] Hinoshita, E., Uchiumi, T., Taguchi, K., Kinukawa, N., Tsuneyoshi, M., Maehara, Y., Sugimachi, K. and Kuwano, M. (2015) Increased Expression of an ATP-Binding Cassette Superfamily Transporter, Multidrug Resistance Protein 2, in Human Colorectal Carcinomas. Clinical Cancer Research, 6, 2401-2407.

[14] Yamasaki, M., Makino, T., Masuzawa, T., Kurokawa, Y., Miyata, H., Takiguchi, S., Nakajima, K., Fujiwara, Y., Matsuura, N., Mori, M., et al. (2011) Role of Multidrug Resistance Protein 2 (MRP2) in Chemoresistance and Clinical Outcome in Oeso- 
phageal Squamous Cell Carcinoma. British Journal of Cancer, 104, 707-713. https://doi.org/10.1038/sj.bjc.6606071

[15] Hirano, T., Kato, H., Maeda, M., Gong, Y., Shou, Y., Nakamura, M., Maeda, J., Yashima, K., Kato, Y., Akimoto, S., et al. (2005) Identification of Postoperative Adjuvant Chemotherapy Responders in Non-Small Cell Lung Cancer by Novel Biomarker. International Journal of Cancer, 117, 460-468.

https://doi.org/10.1002/ijc.21172

[16] Kasahara, K., Fujiwara, Y., Nishio, K., Ohmori, T., Sugimoto, Y., Komiya, K., Matsuda, T. and Saijo, N. (1991) Metallothionein Content Correlates with the Sensitivity of Human Small Cell Lung Cancer Cell Lines to Cisplatin. Cancer Research, 51, 3237-3242.

[17] Dasari, S. and Tchounwou, P.B. (2014) Cisplatin in Cancer Therapy: Molecular Mechanisms of Action. European Journal of Pharmacology, 740, 364-378.

https://doi.org/10.1016/j.ejphar.2014.07.025

[18] Rocha, C.R.R., Silva, M.M., Quinet, A., Cabral-Neto, J.B. and Menck, C.F.M. (2018) DNA Repair Pathways and Cisplatin Resistance: An Intimate Relationship. Clinics, 73, Article No. e478s. https://doi.org/10.6061/clinics/2018/e478s

[19] Deloia, J.A., Bhagwat, N.R., Darcy, K.M., Strange, M., Tian, C., Nuttall, K., Krivak, T.C. and Niedernhofer, L.J. (2012) Comparison of ERCC1/XPF Genetic Variation, mRNA and Protein Levels in Women with Advanced Stage Ovarian Cancer Treated with Intraperitoneal Platinum. Gynecologic Oncology, 126, 448-454.

https://doi.org/10.1016/j.ygyno.2012.05.006

[20] Li, Z., Qing, Y., Guan, W., Li, M., Peng, Y., Zhang, S., Xiong, Y. and Wang, D. (2014) Predictive Value of APE1, BRCA1, ERCC1 and TUBB3 Expression in Patients with Advanced Non-Small Cell Lung Cancer (NSCLC) Receiving First-Line Platinum-Paclitaxel Chemotherapy. Cancer Chemotherapy and Pharmacology, 74, 777-786. https://doi.org/10.1007/s00280-014-2562-1

[21] Villalobos, M., Czapiewski, P., Reinmuth, N., Fischer, J.R., Andreas, S., Kortsik, C., Serke, M., Wolf, M., Neuser, P., Reuss, A., et al. (2018) ERCC1 Assessment in Upfront Treatment with and without Cisplatin-Based Chemotherapy in Stage IIIB/IV Non-Squamous Non-Small Cell Lung Cancer. Medical Oncology, 35, Article No. 106. https://doi.org/10.1007/s12032-018-1169-5

[22] Zhang, Z., Jiang, C. and Hu, L. (2014) Low Expression of Excision Repair Cross-Complementation Group-1 Protein Predicts Better Outcome in Patients with Locally Advanced Nasopharyngeal Cancer Treated with Concurrent Chemoradiotherapy. Tumori Journal, 100, 328-332.

[23] Huang, J., Zhou, Y., Zhang, H., Qu, T., Mao, Y., Zhu, H., Quan, L., Xing, P., Wang, J., He, J., et al. (2013) A Phase II Study of Biweekly Paclitaxel and Cisplatin Chemotherapy for Recurrent or Metastatic Esophageal Squamous Cell Carcinoma: ERCC1 Expression Predicts Response to Chemotherapy. Medical Oncology, 30, Article No. 343. https://doi.org/10.1007/s12032-012-0343-4

[24] Ryu, H., Song, I.C., Choi, Y.S., Yun, H.J., Jo, D.Y., Kim, J.M., Ko, Y.B. and Lee, H.J. (2017) ERCC1 Expression Status Predicts the Response and Survival of Patients with Metastatic or Recurrent Cervical Cancer Treated via Platinum-Based Chemotherapy. Medicine, 96, e9402. https://doi.org/10.1097/MD.0000000000009402

[25] Ma, X., Huang, J., Du, W., Zhuo, H., Zhang, J., Shi, C. and Liu, L. (2015) ERCC1 Plays an Important Role in Predicting Survival Outcomes and Treatment Response for Patients with HNSCC: A Meta-Analysis. Oral Oncology, 51, 483-492.

https://doi.org/10.1016/j.oraloncology.2015.02.094 
[26] Hwang, I.G., Jang, J.S., Do, J.H., Kang, J.H., Lee, G.W., Oh, S.Y., Kwon, H.C., Jun, H.J., Lim, H.Y., Lee, S., et al. (2011) Different Relation between ERCC1 Overexpression and Treatment Outcomes of Two Platinum Agents in Advanced Biliary Tract Adenocarcinoma Patients. Cancer Chemotherapy and Pharmacology, 8, 935-944. https://doi.org/10.1007/s00280-011-1558-3

[27] Shen, D.W., Pouliot, L.M., Hall, M.D., Gottesman, M.M. (2012) Cisplatin Resistance: A Cellular Self-Defense Mechanism Resulting from Multiple Epigenetic and Genetic Changes. Pharmacological Reviews, 64, 706-721.

https://doi.org/10.1124/pr.111.005637

[28] Arts, H.J., Hollema, H., Lemstra, W., Willemse, P.H., De Vries, E.G., Kampinga, H.H. and Van der Zee, A.G. (1999) Heatshock-Protein-27 (hsp27) Expression in Ovarian Carcinoma: Relation in Response to Chemotherapy and Prognosis. International Journal of Cancer, 84, 234-238.

https://doi.org/10.1002/(SICI)1097-0215(19990621)84:3\%3C234::AID-IJC6\%3E3.0. CO;2-9

[29] Liu, J.R., Opipari, A.W., Tan, L., Jiang, Y., Zhang, Y., Tang, H. and Nunez, G. (2002) Dysfunctional Apoptosome Activation in Ovarian Cancer: Implications for Chemoresistance. Cancer Research, 62, 924-931.

[30] Vargas-Roig, L.M., Gago, F.E., Tello, O., Aznar, J.C. and Ciocca, D.R. (1998) Heat Shock Protein Expression and Drug Resistance in Breast Cancer Patients Treated with Induction Chemotherapy. International Journal of Cancer, 79, 468-475. https://doi.org/10.1002/(SICI)1097-0215(19981023)79:5\%3C468::AID-IJC4\%3E3.0. CO;2-Z

[31] Belfi, C.A., Chatterjee, S., Gosky, D.M., Berger, S.J. and Berger, N.A. (1999) Increased Sensitivity of Human Colon Cancer Cells to DNA Cross-Linking Agents after GRP78 Up-Regulation. Biochemical and Biophysical Research Communications, 257, 361-368. https://doi.org/10.1006/bbrc.1999.0472

[32] Huang, T.G., Ip, S.M., Yeung, W.S. and Ngan, H.Y. (2000) Changes in p21WAF1, pRb, Mdm-2, Bax and Bcl-2 Expression in Cervical Cancer Cell Lines Transfected with a p53 Expressing Adenovirus. European Journal of Cancer, 36, 249-256. https://doi.org/10.1016/S0959-8049(99)00247-6

[33] Brozovic, A., Simaga, S. and Osmak, M. (2000) Induction of Heat Shock Protein 70 in Drug-Resistant Cells by Anticancer Drugs and Hyperthermia. Neoplasma, 48, 99-103.

[34] Skvortsov, S., Dudas, J., Eichberger, P., Witsch-Baumgartner, M., Loeffler-Ragg, J., Pritz, C., Schartinger, V.H., Maier, H., Hall, J., Debbage, P., Riechelmann, H., Lukas, P. and Skvortsova, I. (2014) Racl as a Potential Therapeutic Target for Chemo-Radioresistant Head and Neck Squamous Cell Carcinomas (HNSCC). British Journal of Cancer, 110, 2677-2687. https://doi.org/10.1038/bjc.2014.221

[35] Shen, D.W., Liang, X.J., Gawinowicz, M.A. and Gottesman, M.M. (2004) Identification of Cytoskeletal $\left[{ }^{14} \mathrm{C}\right]$ Carboplatin-Binding Proteins Reveals Reduced Expression and Disorganization of Actin and Filamin in Cisplatin-Resistant Cell Lines. Molecular Pharmacology, 66, 789-793. https://doi.org/10.1124/mol.66.4.789

[36] Kurayoshi, M., Yamamoto, H., Izumi, S. and Kikuchi, A. (2007) Post-Translational Palmitoylation and Glycosylation of Wnt5a Are Necessary for Its Signaling. Biochemical Journal, 15, 515-523. https://doi.org/10.1042/BJ20061476

[37] Hanaki, H., Yamamoto, H., Sakane, H., Matsumoto, S., Ohda, H., Sato, A. and Kikuchi, A. (2012) An Anti-Wnt5a Antibody Suppresses Metastasis of Gastric Cancer Cells in Vivo by Inhibiting Receptor-Mediated Endocytosis. Molecular Cancer 
Therapeutics, 11, 298-307. https://doi.org/10.1158/1535-7163.MCT-11-0682

[38] Finne, J. and Mäkelä, P.H. (1985) Cleavage of the Polysialosyl Units of Brain Glycoproteins by a Bacteriophage Endosialidase. Involvement of a Long Oligosaccharide Segment in Molecular Interactions of Polysialic Acid. Journal of Biological Chemistry, 260, 1265-1270. https://doi.org/10.1016/S0021-9258(20)71238-X

[39] Michon, F., Brisson, J.R. and Jennings, H.J. (1987) Conformational Differences between Linear Alpha (2-8)-Linked Homosialooligosaccharides and the Epitope of the Group B Meningococcal Polysaccharide. Biochemistry, 26, 8399-8405. https://doi.org/10.1021/bi00399a055

[40] Kabat, E.A., Liao, J., Osserman, E.F., Gamian, A., Michon, F. and Jennings, H.J. (1988) The Epitope Associated with the Binding of the Capsular Polysaccharide of the Group B. meningococcus and of Escherichia coli K1 to a Human Monoclonal Macroglobulin, IgMNOV. Journal of Experimental Medicine, 168, 699-711. https://doi.org/10.1084/jem.168.2.699

[41] Livingston, B.D., Jacobs, J.L., Glick, M.C. and Troy, F.A. (1988) Extended Polysialic Acid Chains ( $\mathrm{n}>55)$ in Glycoproteins from Human Neuroblastoma Cells. Journal of Biological Chemistry, 263, 9443-9448. https://doi.org/10.1016/S0021-9258(19)76560-0

[42] Muller, D., Stoppini, L., Wang, C. and Kiss, J.Z. (1994) A Role for Polysialylated Neural Cell Adhesion Molecule in Lesion-Induced Sprouting in Hippocampal Organotypic Cultures. Neuroscience, 61, 441-445. https://doi.org/10.1016/0306-4522(94)90424-3

[43] Vutskits, L., Djebbara-Hannas, Z., Zhang, H., Paccaud, J.P., Durbec, P., Rougon, G., Muller, D. and Kiss, J.Z. (2001) PSA-NCAM Modulates BDNF-Dependent Survival and Differentiation of Cortical Neurons. European Journal of Neuroscience, 13, 1391-1402. https://doi.org/10.1046/j.0953-816x.2001.01516.x

[44] Moloney, D.J. and Haltiwanger, R.S. (1999) The O-Linked Fucose Glycosylation Pathway: Identification and Characterization of a Uridine Diphosphoglucose: Fucose-1,3-glucosyltransferase Activity from Chinese Hamster Ovary Cells. Glycobiology, 9, 679-687. https://doi.org/10.1093/glycob/9.7.679

[45] David, B. (2019) Advances in Carbohydrate Chemistry and Biochemistry. Elsevier, Amsterdam, 78.

[46] Ji, J., Li, J., Holmes, L.M., Burgin, K.E., Yu, X., Wagner, T.E. and Wei, Y. (2002) Glycoinositol Phospholipid-Anchored Interleukin 2 but Not Secreted Interleukin 2 Inhibits Melanoma Tumor Growth in Mice. Molecular Cancer Therapeutics, 1, 1019-1024. 


\section{List of Abbreviations}

CTR1: Cu-transporter 1

CTR2: Copper transporter 2

HSPs: Heat shock proteins

MTs: Mitochondrial targeting sequence

NER: Nucleotide excision repair

CDDP: cis-diamminedichloroplatinum

MRPs: Multidrug resistance associated proteins 